\title{
Prophylactic and therapeutic immunomodulatory effects of omega-3 fatty acids in patients with differentiated thyroid cancer scheduled for radioiodine ablation
}

\author{
Zeinab Amirkhani ${ }^{1}$, Mehrosadat Alavi $^{1}$, Mehdi Kalani $^{1}$, Ali Alavianmehr ${ }^{1}$, and Shirin \\ Farjadian $^{1}$ \\ ${ }^{1}$ Shiraz University of Medical Sciences
}

October 13, 2020

\begin{abstract}
Radioactive iodine (RAI)-induced thyrocyte destruction may lead to uncontrolled inflammation. This study was designed to evaluate the prophylactic and therapeutic immunomodulatory effects of omega-3 fatty acids in patients with differentiated thyroid cancer scheduled for RAI ablation. A total of 85 patients were divided into two groups based on radioiodine dosage after thyroidectomy: high-dose with $150 \mathrm{mCi}$ and intermediate-dose with $100 \mathrm{mCi}$. Then patients in each group were randomly divided into three subgroups: G1 with RAI ablation only, G2 treated with omega-3 for 30 days before RAI ablation, and G3 treated with omega-3 for 30 days after RAI ablation. Serum cytokine levels were determined with the cytometric bead assay at different time points. Within-group comparisons showed transient elevation of IL-13 after pretreatment with omega3, significant reductions in Th1+Th17/Th2+Th22 ratio after high-dose RAI ablation, and decreased Th1+Th17/Th2+Th22 and Th1+Th17/Th2+Th9+Th22 ratios after intermediate-dose RAI ablation in G2. Between-group comparisons showed that IL-10 level in G3 was significantly higher than in G1 1 week after high-dose RAI ablation, whereas Th1+Th17/Th2+Th22 and Th1+Th17/Th2+Th9+Th22 ratios were significantly lower in G3 than G2 1 month after intermediate-dose RAI ablation. However, cytokine changes 1 week and 1 month after RAI ablation when adjusted for baseline values showed no differences among groups. Despite observing within-group changes in some cytokines, we found no real changes attributable to a prophylactic or therapeutic anti-inflammatory effect of omega-3. Because of the specific effect of radioactive iodine on thyroid cells, extensive systemic inflammation may not be induced after RAI ablation.
\end{abstract}

\begin{abstract}
Radioactive iodine (RAI)-induced thyrocyte destruction may lead to uncontrolled inflammation. This study was designed to evaluate the prophylactic and therapeutic immunomodulatory effects of omega-3 fatty acids in patients with differentiated thyroid cancer scheduled for RAI ablation. A total of 85 patients were divided into two groups based on radioiodine dosage after thyroidectomy: high-dose with $150 \mathrm{mCi}$ and intermediatedose with $100 \mathrm{mCi}$. Then patients in each group were randomly divided into three subgroups: G1 with RAI ablation only, G2 treated with omega- 3 for 30 days before RAI ablation, and G3 treated with omega-3 for 30 days after RAI ablation. Serum cytokine levels were determined with the cytometric bead assay at different time points. Within-group comparisons showed transient elevation of IL-13 after pretreatment with omega-3, significant reductions in Th1+Th17/Th2+Th22 ratio after high-dose RAI ablation, and decreased Th1+Th17/Th2+Th22 and Th1+Th17/Th2+Th9+Th22 ratios after intermediate-dose RAI ablation in G2. Between-group comparisons showed that IL-10 level in G3 was significantly higher than in G1 1 week after high-dose RAI ablation, whereas Th1+Th17/Th2+Th22 and Th1+Th17/Th2+Th9+Th22 ratios were significantly lower in G3 than G2 1 month after intermediate-dose RAI ablation. However, cytokine changes 1 week and 1 month after RAI ablation when adjusted for baseline values showed no differences among groups.
\end{abstract}


Despite observing within-group changes in some cytokines, we found no real changes attributable to a prophylactic or therapeutic anti-inflammatory effect of omega-3. Because of the specific effect of radioactive iodine on thyroid cells, extensive systemic inflammation may not be induced after RAI ablation.

Keywords: differentiated thyroid cancer, radioiodine ablation, omega-3 fatty acids, cytokines

\section{Introduction}

Thyroid cancer is the most prevalent endocrine malignancy, with an annual incidence of 4.6 per 100,000 and a female-to-male ratio of 3:1 in the USA [1]. Based on the Iran Cancer Data System Registry, the average annual incidence rate of thyroid cancer was reported to be 2.2 per 100,000 between 2004 and 2010 [2]. Thyroid cancers are classified into differentiated (including papillary and follicular) and undifferentiated (anaplastic and medullary) carcinoma. Differentiated thyroid carcinoma (DTC) accounts for about $90 \%$ of all thyroid cancers $[3,4]$. Total or near-total thyroidectomy with lymph node dissection is the main treatment for DTC. Depending on the tumor stage and other criteria that can indicate high risk of recurrence or disease-related mortality, subsequent radioactive iodine $\left({ }^{131} \mathrm{I}, \mathrm{RAI}\right)$ ablation may also be recommended [5]. Like normal thyroid cells, DTC cells have the ability to accumulate iodine - a property that makes it possible to use radioiodine to ablate cancer remnants or treat metastatic lesions. Although RAI ablation is highly effective in the treatment of thyroid cancer, it is relatively toxic and has potential side effects [6].

Cytokines are bioactive molecules which are mainly produced by immune cells, especially helper $\mathrm{T}$ (Th) cells; however, tumor cells are also among the various sources of cytokine secretion [7]. Cytokines play a crucial role in the regulation of immune responses, and cytokine imbalances have been related with different diseases [8]. Changes in cytokines have been reported in patients with different types of cancers in previous studies $[9,10]$, but the data are limited regarding cytokine alterations in thyroid cancer, and particularly after RAI ablation in patients with DTC [11-13].

Radioiodine-induced thyrocyte destruction and the release of large amounts of dangerous molecules such as those in damage-associated molecular patterns, in addition to self-antigens, may lead to uncontrolled inflammation and autoimmune thyroid diseases. Although the induction of specific immune responses against malignant cells may favor cancer inhibition, increased inflammation can contribute to cancer progression [14, 15]. Therefore, modulating excess inflammation in this condition may prevent possible side effects.

Vitamins and dietary supplements have been studied as potential radioprotectors [16]. Vitamins $\mathrm{C}$ and $\mathrm{E}$ are known antioxidants and free radical scavengers [17], and their radioprotective effects have been investigated previously [18]. Omega-3 fatty acids are essential for health and should be provided in the diet. There is some evidence to support an anti-inflammatory effect of omega-3 and its benefits against inflammatory diseases [19], autoimmune diseases [20], asthma [21], and cancers [22, 23]. In light of the increasing incidence of DTC and need for RAI ablation in the management of most patients, which in turn can lead to the dysregulation of immune responses, and considering the role of omega-3 fatty acids in immune regulation, this study was designed to investigate the possible prophylactic and therapeutic immunomodulatory effects of omega-3 in patients with DTC scheduled for RAI ablation.

\section{Materials and methods}

\subsection{Patient selection}

After approval of the study protocol by our University Ethics Committee (approval number: IR.SUMS.MED.REC.1396.76) in compliance with the ethical principles for medical research involving human subjects based on the Declaration of Helsinki, 90 patients with DTC were included in this cross-sectional study from January 2018 to July 2020. All patients had total or near-total thyroidectomy and were referred to the Nuclear Medicine Department at Namazi Hospital affiliated with Shiraz University of Medical Sciences for postsurgical RAI ablation.

After the participants had provided their written informed consent, demographic data including age, sex, family history of thyroid diseases or any syndrome associated with DTC, smoking status, and regular ex- 
ercising (which may affect cytokine profiles) [24] were recorded. Because of the direct effect of depression on cytokine levels [25], depression was also evaluated in each patient with the Persian-language version of the Beck Depression Inventory [26]. Clinical data for each patient were also extracted from their medical records.

The patients were divided into two groups based on radioiodine dosage: high dose with $150 \mathrm{mCi}$ (5.55 GBq) or intermediate dose with $100 \mathrm{mCi}(3.7 \mathrm{GBq})$. Then patients in each group were randomly divided into three subgroups: G1 with RAI ablation only, G2 treated with omega-3 for 30 days before RAI ablation, and G3 treated with omega-3 for 30 days after RAI ablation.

The individual dosages were determined by a nuclear medicine specialist based on the histological grade of each patient's tumor according to the American Thyroid Association Management Guidelines for Adult Patients with DTC [5]. They were scheduled to receive RAI 4 to 6 weeks after surgery after a 10-day low-iodine diet.

Patients who received radioiodine at doses higher than $150 \mathrm{mCi}$, who had been admitted previously for RAI therapy before the study, who had taken omega-3 or other supplements before the study, who had undergone a second thyroid surgery, or who had concurrent chronic inflammatory disease, autoimmune disease or other cancers, were excluded from the study.

\subsection{Sample collection}

The patients in G2 were scheduled to take a soft-gel fish oil capsule of omega-3 fatty acids (Best Formulations Inc., Los Angeles, CA, USA) containing $180 \mathrm{mg}$ eicosapentaenoic acid (EPA) and $120 \mathrm{mg}$ docosahexaenoic acid (DHA) daily for 30 consecutive days before RAI ablation, and those in G3 took the same capsule daily for 30 consecutive days after RAI ablation.

In G2, the first blood sample was collected 1 month before RAI ablation. Then patients started to take omega-3 for 30 days and a second blood sample was collected immediately before RAI ablation. The third and fourth blood samples were collected 1 week and 1 month after RAI ablation, respectively. In G1 and G3, blood samples were collected immediately before RAI ablation as well as 1 week and 1 month after RAI ablation. Serum was isolated from each blood sample and stored at $-20{ }^{\circ} \mathrm{C}$ until cytokine assays, which were done in the same assay batch for all collected samples.

\section{Cytokine assay}

The levels of 13 cytokines belonging to the Th1 (IL-2, TNF- $\alpha$ and IFN- $\gamma$ ), Th2 (IL-4, IL-5, IL-6, IL-10 and IL-13), Th17 (IL-17A, IL-17F and IL-21), Th9 (IL-9) and Th22 (IL-22) families were measured with a commercial multiplex cytometric bead assay kit (BioLegend, San Diego, CA, USA) according to the manufacturer's directions. Briefly, after the desired cytokines in serum samples or standards were captured with a mixture of fluorescein isothiocyanate (FITC)-labeled antibody-coated beads, differentiated by size and fluorescence intensity, a mixture of biotin-conjugated antibodies against each target cytokine and phycoerythrin (PE)-labeled streptavidin were added in succession. The results were visualized with a FACSCalibur flow cytometer (eBioscience, San Diego, CA, USA) and the data were analyzed with FlowCytomix Pro-3.0 software (BioLegend). The minimum detectable concentration of each cytokine in serum with this kit was reported to be $0.9 \mathrm{pg} / \mathrm{mL}$ for IL- $10,0.9 \mathrm{pg} / \mathrm{mL}$ for TNF- $\alpha, 1.1 \mathrm{pg} / \mathrm{mL}$ for IL- 4 , IL- 6 and IL-13, $1.2 \mathrm{pg} / \mathrm{mL}$ for IL-9, $1.3 \mathrm{pg} / \mathrm{mL}$ for IL-2, IL-5 and IL-17F, $1.4 \mathrm{pg} / \mathrm{mL}$ for IFN- $\gamma, 1.8 \mathrm{pg} / \mathrm{mL}$ for IL-17A, $2.2 \mathrm{pg} / \mathrm{mL}$ for IL-22, and $2.3 \mathrm{pg} / \mathrm{mL}$ for IL-21.

\section{Statistical analysis}

Cytokine levels were reported as the mean \pm standard error of the mean. Normal distribution of the data was evaluated with the Shapiro-Wilk test, and because the data did not show a normal distribution, nonparametric statistical tests were used. In each high-dose or intermediate-dose RAI ablation group, correlations between cytokine levels before any intervention with age, depression score, tumor size and thyroglobulin serum level were calculated with Spearman's rank test, and correlations with sex, smoking status, exercise 
habits, family history of thyroid diseases, and lymph node involvement were calculated with the MannWhitney U test. The distribution of demographic and clinicopathological characteristics in subgroups G1, G2 and G3 in the high-dose or intermediate-dose RAI ablation groups was examined separately with the chi-squared or Kruskal-Wallis test. The Wilcoxon test was used to calculate differences in cytokine levels between each pair of related samples in each subgroup at different time points. The Kruskal-Wallis test was used to compare cytokine levels among G1, G2 and G3 1 week and 1 month after RAI ablation with 100 $\mathrm{mCi}$ or $150 \mathrm{mCi}$. This test was also used to compare cytokine changes in G1, G2 and G3 in each of the high-dose or intermediate-dose RAI ablation groups with adjustment to their baseline values. All statistical analyses were done with SPSS v. 22 software, and a two-tailed P value ¡0.05 was considered statistically significant. Bonferroni correction was used for multiple comparison tests. GraphPad PRISM 6 was used to create graphical displays.

\section{Results}

A total of 90 patients with DTC were initially enrolled in the study. Five patients were excluded during the study: 3 because of noncompliance with the treatment and 2 because of a missed appointment for blood sampling. A total of 85 patients completed the study: 11 males and 74 females, with mean age $37.5 \pm 11.9$ years (range 18-67 years). All patients were nonsmokers, and none of them reported exercising regularly. None of them had a family history of thyroid cancer, and none had any other malignancies, active infections, chronic inflammatory or autoimmune diseases.

The characteristics of patients in different subgroups are summarized in Table 1. Our results showed negative associations of age with IL-2, IL-17A, IL-17F levels and with all cytokine ratios, and positive associations of sex with IL-17F level, as well as between depression score and IL-9 and IL-21 level before any intervention in patients scheduled for high-dose RAI ablation. We also found negative associations of depression score with Th17/Th2 and Th1+Th17/Th2+Th22 ratios, and positive associations of tumor size with IL-9, IL-17A, TNF- $\alpha$ levels and Th17/Th2 ratio before any intervention in patients scheduled for intermediate-dose RAI ablation (Table S1).

The cytokine levels at different time points in patients with DTC who had RAI ablation with or without omega-3 treatment are compared in Figure. 1. In G1 patients, IL-10 level was significantly increased $(\mathrm{P}=0.01) 1$ month compared to 1 week after high-dose RAI ablation, and IL-17F and IL-21 levels were decreased ( $\mathrm{P}=0.008$ and $\mathrm{P}=0.041$, respectively) 1 week after intermediate-dose RAI ablation compared to the time point immediately before RAI ablation. There were no changes in Th subset cytokines or ratios among the three time points in this group (Table S2).

In G2 patients (pretreated with omega-3), despite the absence of changes in the level of any of the cytokines or Th subset cytokines, Th1+Th17/Th2+Th22 ratio was significantly decreased $(\mathrm{P}=0.029) 1$ week after high-dose RAI ablation compared to immediately before ablation. In G2 patients with intermediatedose RAI, IL- 4 was significantly reduced $(\mathrm{P}=0.001) 1$ month after ablation compared to the time point immediately before ablation. IL-13 showed a significant but transient increase $(\mathrm{P}=0.042)$ after pretreatment with omega-3 but returned to the baseline level after 1 month, whereas Th1+Th17/Th2+Th22 and Th1+Th17/Th2+Th9+Th22 ratios were significantly decreased ( $\mathrm{P}=0.042$ for each) 1 week after RAI ablation compared to immediately before ablation (Table S3).

In G3 patients who received high-dose RAI and post-treatment with omega-3, IL-6 ( $\mathrm{P}=0.033$ ), IL-13 $(\mathrm{P}=0.032)$ and $\mathrm{Th} 2$ cytokines $(\mathrm{P}=0.027)$ were significantly increased 1 week after treatment. The significant increase in Th2 was maintained for up to 1 month after treatment $(\mathrm{P}=0.027)$; however, IL-4 level decreased significantly $(\mathrm{P}=0.042)$ at 1 month compared to 1 week after the intervention. Post-treatment with omega-3 in patients who received intermediate-dose RAI showed no remarkable changes in any of the cytokines tested, or in Th subset cytokines or ratios (Table S4).

Between-group comparisons of cytokine levels are summarized in Figure 2. As shown, IL-10 levels in G2 and G3 were higher than G1 1 week after high-dose RAI ablation, although only the difference between G3 and G1 was significant $(\mathrm{P}=0.013)$. Notable decreases in Th1+Th17/Th2+Th22 $(\mathrm{P}=0.007)$ and 
Th1+Th17/Th2+Th9+Th22 ratios $(\mathrm{P}=0.01)$ were seen in G3 compared to G2 1 month after intermediatedose RAI ablation, although these ratios were similar in G1 and G2.

In G3, baseline IL-4 level was significantly lower than in G1 and G2 before high-dose RAI ablation ( $\mathrm{P}=0.0007$ and $\mathrm{P}=0.0096$, respectively), even in comparison to $\mathrm{G} 2$ before any intervention ( $\mathrm{P}=0.0007$ and $\mathrm{P}=0.0133$, respectively). Baseline Th2 level in G3 was lower than in G2 (but similar to the level in G1) before high-dose RAI ablation $(\mathrm{P}=0.0096)$. In $\mathrm{G} 3$, the baseline Th17/Th2 ratio was also significantly lower than in $\mathrm{G} 2$ before intermediate-dose RAI ablation (Table S5). Despite these differences, comparisons of the changes in cytokine levels 1 week and 1 month after RAI ablation, when adjusted to their baseline values, showed no differences among the three groups (Table S6).

\section{Discussion}

The increasing incidence of thyroid cancer requires serious attention to early diagnosis and better patient management. The progression of cancers is affected by the cytokine network, and it is well established that Th1 cytokines support antitumor immune responses whereas Th2 cytokines facilitate tumor progression due to the downmodulation of cell-mediated immunity [27]. In addition, some findings show that inflammation is a critical component in tumor progression; in this connection a strong link between DTC and inflammation has been reported [28], and was attributed to inflammatory cytokines such as TNF, IL-1, IL-6 and IL-17F produced not only by tumor-infiltrating leukocytes but also by tumor cells themselves [29].

Despite the direct or inverse correlations of some cytokines with age, sex, depression score or tumor size (Supplementary Table 1), because our patients were almost uniformly distributed among G1, G2 and G3 in each of the two main groups scheduled for high-dose or intermediate-dose RAI ablation, none of the demographic or clinicopathologic features seemed to have a confounding effect on cytokines (Table 1).

Therapeutic procedures including radiotherapy also affect cytokines, which in turn may affect immune responses against malignant cells [9, 30, 31]. Jones et. al. reported transient increases in IL-4, IL-6 and IL-10 after RAI therapy for Graves' disease [30]. Ozata et al. also found a significant increase in IL-6 2 months after RAI therapy in patients with DTC, in the absence of changes in TNF- $\alpha$ levels [11]. Georgakilas et al. [32] and Yahyapour et al. [33] provided some evidence of radiation-induced inflammation. The results of the present study disclosed increased levels of IL-10 1 month after high-dose RAI ablation. Although increased IL-10 downmodulates immune responses against malignant cells, it may also prevent metastases by reducing inflammation. Our results, however, are inconsistent with findings reported by Demir et al., who observed some cellular evidence of increased systemic inflammation 2 months after RAI ablation with 100 or $150 \mathrm{mCi}$ in patients with DTC [34]. We also observed significant reductions in IL-17F and IL-21 levels 1 week after intermediate-dose RAI ablation in our patients (G1: Fig. 1; Table S2). Although IL-17F and IL-21 are both proinflammatory cytokines [35] produced mainly by Th17 cells, we found no significant decrease in overall Th17-related cytokins - a result that can be explained by the nonsignificant increase in IL-17A. Decreased IL-17F and IL-21 after RAI ablation may prevent tumor invasion by reducing inflammation. Our results are consistent with Zhang et al., who reported an elevated frequency of Th17 cells in peripheral blood and their related serum cytokines in patients with DTC compared to healthy controls, a significant decline in these markers 2 week after ${ }^{131}$ I therapy, and a return to normal levels after 3 months [36]. It is worth noting that, considering the complex interactions among cytokines within an extensive network, increased IL-10 alone or decreased IL-17 and IL-21 without affecting overall cytokine balance may not result in any specific biological effect [37]

There is evidence in support of the beneficial effects of omega- 3 on recovery in patients who undergo chemotherapy, radiotherapy, or both [38]. Selective cytotoxicity of omega-3 against cancer cells was reported by D'Eliseo et al., who suggested prescribing omega-3 in combination with conventional anticancer therapies [39]. To discover whether omega-3 had any beneficial prophylactic effect in patients treated with RAI ablation, a subgroup of our patients with DTC was pretreated with omega-3 for 30 days. Although we found no change in the level of any cytokines or Th subset cytokines, Th1+Th17/Th2+Th22 ratio was significantly decreased 1 week after high-dose RAI ablation, which indicated an overall decline in inflammatory 
cytokines or an enhancement of anti-inflammatory cytokines. Although our findings should be considered preliminary, these changes may curtail high-grade tumor progression. In patients who were pretreated with omega-3 and then received intermediate-dose RAI, IL-13 was clearly increased immediately after the course of omega-3, whereas IL-4 was substantially decreased 1 month after RAI ablation. Despite the nonsignificant increases in Th2, Th1+Th17/Th2+Th22 and Th1+Th17/Th2+Th9+Th22 ratios after omega-3 consumption, these ratios were lower 1 week after RAI ablation (G2: Fig. 1; Table S3). This might be explained by the decrease in the sum of minor changes in Th1- and/or Th17-related cytokines, or by the increase in the sum of minor changes in Th9-, Th22- or Th2-related cytokines. Our results showed that omega-3 prophylaxis in patients with DTC shifted the overall cytokine balance towards a temporary increase in cellular immunity immediately before RAI ablation, which is critical for the effective removal of malignant cells, and then redirection of the overall cytokine balance toward a reduction in inflammation 1 week after RAI ablation, which was potentially helpful in preventing metastases in our patients with high-dose and intermediate-dose RAI ablation.

To investigate the therapeutic effects of omega-3 in patients treated with RAI ablation, a group of patients with DTC was post-treated with omega-3 for 30 days after ablation. Our results showed significant elevations in IL-4, IL-6, IL-13 and consequently Th2 cytokine levels 1 week after high-dose RAI ablation. Despite successive decreases in IL-4 and IL-13 levels after 1 month, Th2 elevation was maintained for up to 1 month, which can be explained by the increasing trend in IL-6 at 1 month after treatment (G3: Fig. 1; Table S4), even though Th2 elevation had no remarkable effect on cytokine ratios. Although Th2 activity prevents Th1-mediated antitumor responses, IL-6, a Th2 cytokine which is also produced by other cells, is a proinflammatory cytokine which can lead to extensive tumor expansion [40]. In contrast to our results based on the increase in Th2 cytokines 1 week after RAI ablation with $150 \mathrm{mCi}$ in combination with omega3, Simonovic et al. reported reduced Th2 cytokines in the supernatant of phytohemagglutinin-stimulated peripheral blood cells of patients with DTC 1 week after treatment with $100 \mathrm{mCi}$ RAI [12]. This inconsistancy may be viewed as an effect of omega-3 in the present study. It may also be due to the difference in the doses of RAI used in these two studies, or the explanation may lie in more complex interactions among various factors in the present in vivostudy compared to the in vitro study by Simonvic et al. The elevation in IL-10 in G1 1 month after high-dose RAI ablation, which is assumed to be one of the beneficial effects of RAI, was greater in G2 and G3, possibly owing to omega-3 consumption.

The transient reduction in IL-17F and IL-21 in G1 1 week after intermediate-dose RAI ablation can also be considred a favorable effects of RAI, although both levels returned to baseline values after 1 month. However, these two cytokines showed no significant chenges in G2 or in G3. Unlike G3, Th1+Th17/Th2+Th22 and Th1+Th17/Th2+Th9+Th22 ratios were decreased in G2 (Fig. 2; Table S5).

Due to the difference in the baseline values of some cytokines before any intervention (Table S5), to further compare cytokine behaviors among subgroups, between-group differences were analyzed with the baseline value in each subgroup as a reference. We found no difference in any of the cytokines tested, or in Th subset cytokines and ratios, among the three subgroups 1 week and 1 month after RAI ablation when cytokine levels were adjusted to their baseline levels (Table S6).

Despite observing within-group changes in some cytokines, we did not find evidence from the changes across groups that could indicate a prophylactic or therapeutic anti-inflammatory effect of omega-3. Due to the specific accumulation of radioactive iodine in thyroid cells, extensive systemic inflammation may not be induced after RAI ablation. To investigate the immunomodulatory effect of omega-3 in reducing the side effects of radiotherapy, other solid malignancies treated with non-targeted radiotherapy or hematologic malignancies treated with total body irradiation should be investigated.

The main strengths of this study were the inclusion of a large number of patients with DTC who received different doses of RAI, and the simultaneous evaluation of 13 cytokines belonging to the main Th subsets with a precise quantitative method. One limitation of our study was the short-term follow-up; longer follow-up periods are needed to evaluate the efficacy of omega-3 in strengthening the immune profile and improving the prognosis in patients with cancer. All patients in the present study received the same dose of 
omega-3 for a period of 30 days before or after RAI ablation. Long-term studies of omega-3 consumption starting some time before radiotherapy and continuing after therapy, with different doses of omega-3 in combination with omega- 6 and omega-9 for different durations, may shed additional light on the potential immune regulatory benefits of this dietary supplementation. Another potential limitation of our study was the lack of a placebo-controlled group. Olive oil has been used as a placebo in similar studies, but because of its potentially immunomodulatory effects, it may produce misleading results [41, 42]. To clarify points which remain obscure, future analyses should focus on intracellular cytokine production by different $\mathrm{T}$ cell subsets in peripheral blood, tumor-infiltrating T cells, tumor-draining lymph nodes, and tumor cells in patients with DTC who receive a combination of RAI and omega-3.

\section{Conclusion}

Despite observing within-group changes in some cytokines, we found no real changes attributable to a prophylactic or therapeutic anti-inflammatory effect of omega-3. Because of the specific effect of radioactive iodine on thyroid cells, extensive systemic inflammation may not be induced after RAI ablation. To investigate the potential for immunomodulatory effects of omega-3 to reduce the side effects of radiotherapy, other solid malignancies with non-targeted radiotherapy, or hematologic malignancies treated with total body irradiation, should be investigated.

\section{Acknowledgments}

The authors thank Professor P. Jafari for his critical help with statistical analyses, and K. Shashok (AuthorAID in the Eastern Mediterranean) for improving the use of English in the manuscript.

\section{Funding}

This work was financially supported by a grant from the National Institute for Medical Research Development (NIMAD grant number: 958687).

\section{Ethical approval}

All procedures involving human participants were approved by the Ethics Committee of Shiraz University of Medical Sciences (Decision no: IR.SUMS.MED.REC.1396.76) in accordance with the 1964 Helsinki Declaration and its later amendments or comparable ethical standards.

\section{Declaration of Competing Interest}

The authors declare no conflict of interest.

\section{References}

1. James, B.C., et al., An update in international trends in incidence rates of thyroid cancer, 1973-200\%. Cancer Causes Control, 2018. 29 (4-5): p. 465-473.

2. Safavi, A., et al., Thyroid Cancer Epidemiology in Iran: a Time Trend Study. Asian Pac J Cancer Prev, 2016. 17 (1): p. 407-12.

3. Cabanillas, M.E., D.G. McFadden, and C. Durante, Thyroid cancer. Lancet, 2016. 388 (10061): p. 2783-2795.

4. Kent, W.D., et al., Increased incidence of differentiated thyroid carcinoma and detection of subclinical disease. CMAJ, 2007.177 (11): p. 1357-61.

5. Haugen, B.R., et al., 2015 American Thyroid Association Management Guidelines for Adult Patients with Thyroid Nodules and Differentiated Thyroid Cancer: The American Thyroid Association Guidelines Task Force on Thyroid Nodules and Differentiated Thyroid Cancer. Thyroid, 2016. 26 (1): p. 1-133.

6. Fard-Esfahani, A., et al., Adverse effects of radioactive iodine-131 treatment for differentiated thyroid carcinoma. Nucl Med Commun, 2014. 35 (8): p. 808-17. 
7. Dunlop, R.J. and C.W. Campbell, Cytokines and advanced cancer.J Pain Symptom Manage, 2000. 20 (3): p. 214-32.

8. Gulati, K., et al., Cytokines and their role in health and disease: a brief overview. MOJ Immunol, 2016. 4 (2): p. 00121.

9. King, J., H. Mir, and S. Singh, Association of Cytokines and Chemokines in Pathogenesis of Breast Cancer. Prog Mol Biol Transl Sci, 2017. 151 : p. 113-136.

10. Baier, P.K., et al., Cytokine expression in colon carcinoma.Anticancer Res, 2005. 25 (3B): p. 2135-9.

11. Özata, M., et al., Effect of radioiodine therapy on several hematological and immune parameters in patients with differentiated thyroid carcinoma. Turk J Endocrinol Metab 2000. 2 : p. 45-50

12. Simonovic, S.Z., et al., Cytokine production in peripheral blood cells of patients with differentiated thyroid cancer: elevated Th2/Th9 cytokine production before and reduced Th2 cytokine production after radioactive iodine therapy. Cancer Immunol Immunother, 2015.64 (1): p. 75-82.

13. Zhang, L., et al., Effects of iodine-131 radiotherapy on Th17/Tc17 and Treg/Th17 cells of patients with differentiated thyroid carcinoma. Exp Ther Med, 2018. 15 (3): p. 2661-2666.

14. Riley, A.S., et al., The effect of radioiodine treatment on the diseased thyroid gland. Int J Radiat Biol, 2019. 95 (12): p. 1718-1727.

15. Du, W., et al., Iodine-131 therapy alters the immune/inflammatory responses in the thyroids of patients with Graves' disease. Experimental and Therapeutic Medicine, 2017. 13 (3): p. 1155-1159.

16. Mathew, D., et al., Ascorbic acid monoglucoside as antioxidant and radioprotector. J Radiat Res, 2007. 48 (5): p. 369-76.

17. Rosario, P.W., K.C. Batista, and M.R. Calsolari,Radioiodine-induced oxidative stress in patients with differentiated thyroid carcinoma and effect of supplementation with vitamins $C$ and $E$ and selenium (antioxidants). Arch Endocrinol Metab, 2016. 60 (4): p. 328-32.

18. jafari E, A.M., Zal F., The evaluation of protective and mitigating effects of vitamin $C$ against side effects induced by radioiodine therapy. Radiation and Environmental Biophysics 2017.2 : p. 1-8.

19. Iverson, C., et al., Omega-3-carboxylic acids provide efficacious anti-inflammatory activity in models of crystal-mediated inflammation. Sci Rep, 2018. 8 (1): p. 1217.

20. Berbert, A.A., et al., Supplementation of fish oil and olive oil in patients with rheumatoid arthritis. Nutrition, 2005.21 (2): p. 131-6.

21. Farjadian, S., et al., Effects of omega-3 fatty acids on serum levels of T-helper cytokines in children with asthma. Cytokine, 2016.85 : p. 61-6.

22. Fabian, C.J., B.F. Kimler, and S.D. Hursting, Omega-3 fatty acids for breast cancer prevention and survivorship. Breast Cancer Res, 2015. 17 : p. 62.

23. Terry, P.D., J.B. Terry, and T.E. Rohan, Long-Chain (n-3) Fatty Acid Intake and Risk of Cancers of the Breast and the Prostate: Recent Epidemiological Studies, Biological Mechanisms, and Directions for Future Research. J Nutr, 2004. 134(12 Suppl) : p. 3412-19.

24. Daloee, M.H., et al., Impact of Cigarette Smoking on Serum Pro- and Anti-Inflammatory Cytokines and Growth Factors. Am J Mens Health, 2017. 11 (4): p. 1169-1173.

25. Kiecolt-Glaser, J.K. and R. Glaser, Depression and immune function: central pathways to morbidity and mortality. J Psychosom Res, 2002. 53 (4): p. 873-6.

26. Ghassemzadeh, H., et al., Psychometric properties of a Persian-language version of the Beck Depression Inventory-Second edition: BDI-II-PERSIAN. Depress Anxiety, 2005. 21 (4): p. 185-92. 
27. Kidd, P., Th1/Th2 balance: the hypothesis, its limitations, and implications for health and disease. Altern Med Rev, 2003.8 (3): p. 223-46.

28. Muzza, M., et al., The tight relationship between papillary thyroid cancer, autoimmunity and inflammation: clinical and molecular studies. Clin Endocrinol (Oxf), 2010. 72 (5): p. 702-8.

29. Coussens, L.M. and Z. Werb, Inflammation and cancer. Nature, 2002. 420 (6917): p. 860-7.

30. Jones, B.M., C.C. Kwok, and A.W. Kung, Effect of radioactive iodine therapy on cytokine production in Graves' disease: transient increases in interleukin-4 (IL-4), IL-6, IL-10, and tumor necrosis factor-alpha, with longer term increases in interferon-gamma production. J Clin Endocrinol Metab, 1999. 84 (11): p. 4106-10.

31. Lumachi, F., S.M. Basso, and R. Orlando, Cytokines, thyroid diseases and thyroid cancer. Cytokine, 2010. 50 (3): p. 229-33.

32. Georgakilas, A.G., et al., Emerging molecular networks common in ionizing radiation, immune and inflammatory responses by employing bioinformatics approaches. Cancer Lett, 2015. 368 (2): p. 164-72.

33. Yahyapour, R., et al., Radiation-induced inflammation and autoimmune diseases. Mil Med Res, 2018. 5 (1): p. 9.

34. Demir, Y., et al., Temporary changes in neutrophil-to-lymphocyte, platelet-to-lymphocyte ratios, and mean platelet volume reflecting the inflammatory process after radioiodine therapy. Nucl Med Commun, 2016. 37 (4): p. 393-8.

35. Wojdasiewicz, P., L.A. Poniatowski, and D. Szukiewicz, The role of inflammatory and anti-inflammatory cytokines in the pathogenesis of osteoarthritis. Mediators Inflamm, 2014. 2014 : p. 561459.

36. Zhang, L., et al., Effects of iodine-131 radiotherapy on Th17/Tc17 and Treg/Th17 cells of patients with differentiated thyroid carcinoma. Experimental and Therapeutic Medicine, 2018. 15 (3): p. 2661-2666.

37. Yamashita, T., et al., Serum cytokine profiles are altered in patients with progressive infantile hemangioma. Biosci Trends, 2018.12 (4): p. 438-441.

38. de Aguiar Pastore Silva, J., M. Emilia de Souza Fabre, and D.L. Waitzberg, Omega-3 supplements for patients in chemotherapy and/or radiotherapy: A systematic review. Clin Nutr, 2015. 34 (3): p. 359-66.

39. D'Eliseo, D. and F. Velotti, Omega-3 fatty acids and cancer cell cytotoxicity: implications for multitargeted cancer therapy. J Clin Med, 2016. 5 (2): p. 15.

40. Unver, N. and F. McAllister, IL-6 family cytokines: Key inflammatory mediators as biomarkers and potential therapeutic targets. Cytokine Growth Factor Rev, 2018. 41 : p. 10-17.

41. Brunelleschi, S., et al., Minor polar compounds extra-virgin olive oil extract (MPC-OOE) inhibits NFkappa B translocation in human monocyte/macrophages. Pharmacol Res, 2007. 56 (6): p. 542-9.

42. Fukumitsu, S., et al., Anti-inflammatory and anti-arthritic effects of pentacyclic triterpenoids maslinic acid through NF-kappaB inactivation. Mol Nutr Food Res, 2016. 60 (2): p. 399-409.

\section{Figure legends:}

Fig. 1. Cytokine changes in patients with DTC at different time points after RAI ablation preceded or followed by omega-3 fatty acid treatment. * indicates $\mathrm{P}<0.05$, ** indicates $\mathrm{P}<0.01$. Detailed information is presented in Tables S2-S4.

Fig. 2 Between-group comparisons of cytokines in patients with DTC 1 week and 1 month after RAI ablation: G1 with RAI ablation only, G2 treated with omega-3 fatty acids for 30 days before RAI ablation, and G3 treated with omega-3 for 30 days after RAI ablation. * indicates $\mathrm{P}<0.05$. Detailed information is presented in Table S5. 


\section{Hosted file}

Table 1.pdf available at https://authorea.com/users/366814/articles/486448-prophylacticand-therapeutic-immunomodulatory-effects-of-omega-3-fatty-acids-in-patients-withdifferentiated-thyroid-cancer-scheduled-for-radioiodine-ablation
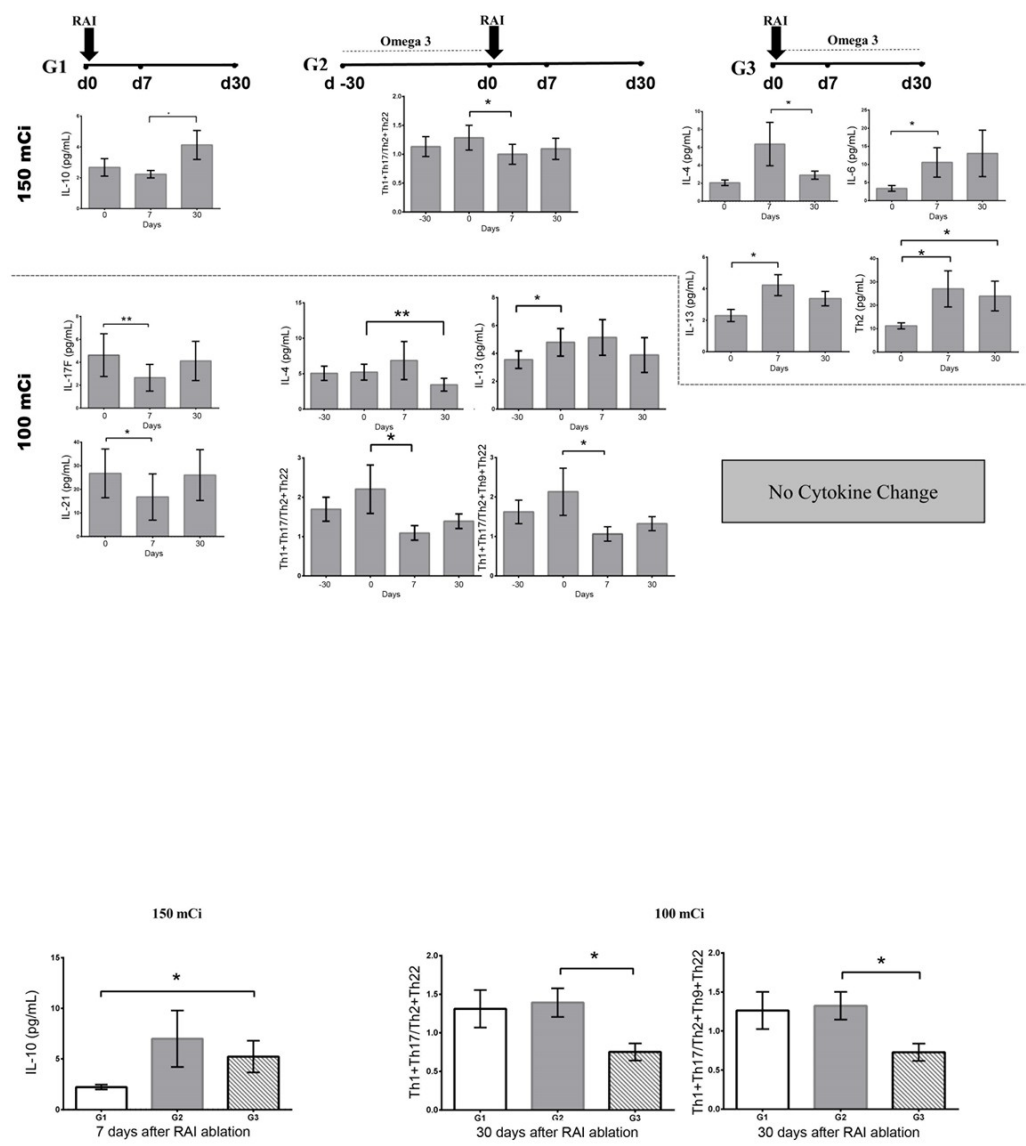